\title{
Interferometric apodization of rectangular apertures
}

\section{Application to stellar coronagraphy}

\author{
C. Aime, R. Soummer, and A. Ferrari \\ UMR 6525 Astrophysique, Faculté des Sciences, Université de Nice Sophia Antipolis, Parc Valrose, \\ 06108 Nice Cedex 2, France \\ e-mail: Claude.Aime@unice.fr, Andrea.Ferrari@unice.fr
}

Received 17 July 2001 / Accepted 20 August 2001

\begin{abstract}
We describe the principle of an apodization technique for rectangular apertures that can be implemented using a Michelson or a Mach-Zender interferometer. Using several interferometers, any integer power of cosine functions can be obtained. The technique is considered for application to the Apodized Square Aperture (ASA) concept recently proposed by Nisenson \& Papaliolios (2001). Simple analytic expressions for the Point Spread Functions of such apodized apertures are given. For a cosine to the power $N$ apodization, the resulting focal plane amplitude is simply the sum of $N+1$ weighted and shifted sine cardinal functions. The interest of such apodized apertures for coronagraphy is investigated. It appears that these apodization functions are very efficient provided that only a central part of the cosine-arch is used. Analytic expressions are derived for the residual amplitude left in an image of the aperture after the coronagraphic experiment. Best results are obtained with a cosine squared apodization.
\end{abstract}

Key words. instrumentation: high angular resolution - techniques: interferometric - techniques: high angular resolution - telescopes - stars: planetary systems

\section{Introduction}

One of the most ambitious objectives of present astronomy is the direct detection of an Earth-like planet orbiting a nearby star, and the spectral analysis of its atmosphere to search for life. At first sight, the required angular resolution is not that great: seen at 10 parsec, the angular separation between Earth and Sun is 0.1 arcsec. There are about 100 to 200 target stars (F, G, K, M) within that distance (TPF Science Working Group 1999). The main difficulties come from the brightness ratio between the planet and its parent star. This ratio, of the order of $10^{9}$ in the visible, presents its most favorable value of about $10^{6}$ in the infrared near $10 \mu \mathrm{m}$. The infrared range of wavelength is also favorable for the detection of molecular absorption bands such as $\mathrm{H}_{2} \mathrm{O}, \mathrm{CO}_{2}$ and $\mathrm{O}_{3}$ (Léger et al. 1999).

The received flux from a planet located at 3 or 4 Airy discs from the star is about $10^{3}$ to $10^{6}$ times fainter than the average level of star-diffracted light and the photon

Send offprint requests to: R. Soummer,

e-mail: Remi.Soummer@unice.fr noise will prevent the direct observation of the planet in realistic observing times.

Several techniques have been recently studied to cancel as best possible the star diffracted light (Roddier \& Roddier 1997; Gay \& Rabbia 1996; Baudoz et al. 2000a,b; Abe et al. 2001; Rouan et al. 2000). They can be roughly classified in three groups of methods, one referring to Nulling interferometry (Bracewell 1978; Mennesson \& Mariotti 1997; Angel \& Woolf 1997), the second to direct imaging using coronagraphy and the last to apodization (Nisenson \& Papaliolios 2001). Nulling interferometry has lead to the Darwin project (Leger 1993; Leger et al. 1996), while coronagraphic techniques are presently under study for TPF (TPF Science Working Group 1999; Guyon \& Roddier 2000; Boccaletti et al. 2000; Pedretti et al. 2000; Aime et al. 2001).

Coronagraphy, invented by Lyot $(1930,1939)$ for the observation of the solar corona, is the first recognized technique to provide direct imaging capabilities of exoplanets (Bonneau et al. 1975; Malbet 1996; Watson et al. 1991). The technique has been recently improved by the use of a $\pi$ Phase Mask (PM) instead of the Lyot's opaque mask. (Roddier \& Roddier 1997; Guyon et al. 1999). To obtain 
the required reduction factors necessary for telluric planet detection, coronagraphy must be coupled with an apodization of the entrance aperture. In that case, reduction factors as large as $10^{7}$ have been reported for the monochromatic case (Guyon \& Roddier 2000). This gain is obtained at the expense of a small loss of transmission.

Recently, Nisenson \& Papaliolios (2001) proposed the alternative concept of Apodized Square Aperture (ASA), based on the elegant idea introduced by Watson et al. (1991) to take advantage of the rapid drop of the diffracted light along the diagonal direction for a square aperture. Provided that the adequate apodizer is realized, these authors have shown that an ASA can reach the required dynamic range for planet detection without coronagraphy. They describe transmission apertures with several shapes of apodizations (sonine, cosine, or squared cosine) for which the overall transmission factors are of the order of $15 \%$.

The work proposed in this paper originates from the search of an apodization method based on an interference process which may be more effective than the classical one using amplitude transmission masks. Its principle and possible optical implementation are given in Sect. 2. The method makes it possible to obtain any power of cosine apodizations such as the ones considered for the ASA concept. For these apodizations, we give in Sect. 3 the analytical expressions for the PSF observed at the focus of the telescope.

The same kind of cosine apodization is analyzed for its use in stellar coronagraphy in Sect. 4: the cosine shape appears to be very efficient, provided that only a fraction of the cosine arch is used (larger for Lyot's mask than for R\&R's mask). Section 5 examines the chromatic effets of the technique. Conclusion are given in Sect. 6. As we shall see, the proposed interferometric apodization technique appears to be a versatile solution.

\section{Interferometric apodization of rectangular apertures}

The purpose of apodization is to obtain a redistribution of the energy in the diffraction pattern, reducing the energy in the wings of the PSF. In Signal Processing, the problem has been extensively studied for the spectral analysis of temporal signals (Papoulis 1981; Harris 1978). In Optics, this effect can be obtained with appropriate modifications of the pupil function. A large review of apodization techniques was made by Jacquinot \& Roizen-Dossier (1964). These authors also suggested to illuminate the pupil with an interference pattern in a spectroscopic system for better slit illumination, but they abandoned the idea. We reconsider this idea herein and give below the principle of the technique.

For the ASA concept, and more generally for an apodized rectangular aperture, the pupil transmission and its corresponding focal plane diffraction can be written as separate functions of $x$ and $y$. This property can be used to describe the technique for a one-dimensional telescope.

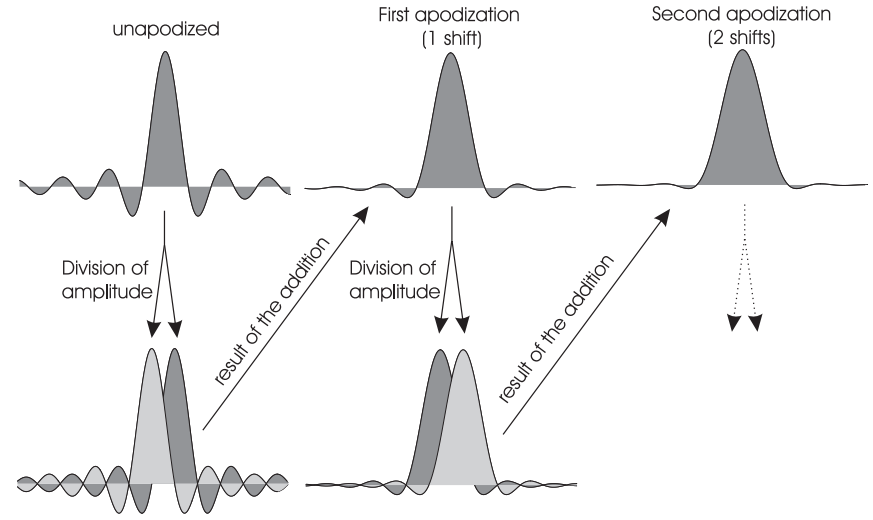

Fig. 1. Illustration of the apodization technique in the focal plane. Top left: unapodized amplitude split into two shifted amplitude (bottom left). The result of this coherent addition is the first-apodized amplitude (top center). This approach can be iteratively reproduced: the split and shifted amplitudes (bottom center) and second-apodized amplitude (top right).

The generalization to a two-dimensional rectangular or square aperture is straightforward.

\subsection{Principle of the technique}

For a one-dimensional telescope of width $L$, the pupil $P(x)$ may be simply written as the window function $\Pi(x / L)$ equal to 1 for $-\frac{L}{2}<x<\frac{L}{2}$ and 0 otherwise.

In the focal plane, the normalized monochromatic amplitude impulse response $\Psi(x)$ is proportional to its Fourier transform:

$\Psi(x)=\frac{\sin \pi L x}{\pi L x}$

where $x$ is the angular position in the focal plane and $L$ must be expressed in units of wavelength $\lambda$.

The proposed apodization technique is very simple. Let us assume that by some means, we are able to split this amplitude $\Psi(x)$ into two identical waves and shift one of them by $\frac{1}{L}$. The minima of the first wave then correspond to the maxima of the second and provided that we maintain the coherence, we obtain the addition of the two amplitudes. Outside the central part of the PSF, positive and negative values approximately cancel each other (Fig. 1). The amplitude of the wings of the PSF is then reduced with, of course, the drawback of an enlarged central peak.

The resulting amplitude remains oscillatory with the same distance $1 / L$ between zeros. We can iteratively reproduce this approach: several identical optical systems can be used successively to produce the shifts and obtain the different degrees of apodization. The procedure is schematized in Fig. 1.

These successive apodizations can be considered as a direct superposition of several shifted amplitudes with appropriate coefficients. The second-order apodization is equivalent to the addition of three shifted amplitudes with coefficients $(1,2,1)$. The third apodization is a fouramplitude addition, with coefficients $(1,3,3,1)$. It can 


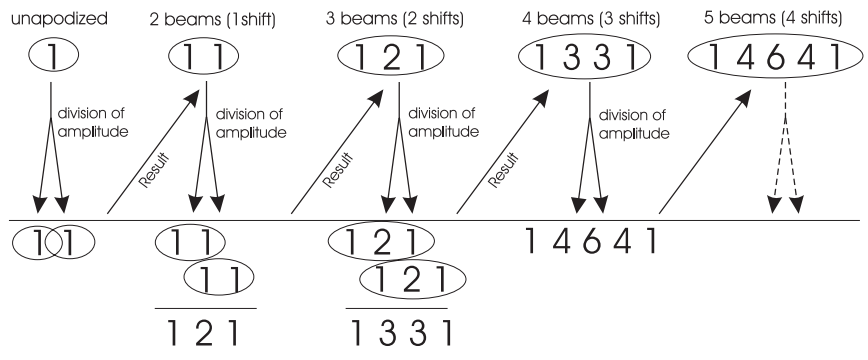

Fig. 2. Illustration of the amplitude coefficients for successive apodizations. The encircled numbers corresponds to the amplitude coefficients. The first apodization corresponds to a 2 beam interference, the second apodization to a 3 beam interference with coefficients 1, 2, 1 for the amplitude. Amplitude coefficients are given by Pascal's Triangle.

be easily shown from the illustration that the amplitude coefficients are given by Pascal's triangle (Fig. 2).

\subsection{Possible optical realization}

A shift of the two identical amplitudes in the focal plane corresponds to a slight tilt of the wavefronts at the pupil plane. This is equivalent to considering localized fringes of equal thickness on the pupil.

This can be easily achieved with an interferometer by division of amplitude, such as the Michelson or the Mach-Zendher interferometer, setting images of the entrance aperture on the interferometer mirrors of the interferometer and slightly tilting a mirror to introduce a thin wedge-shaped film.

The required shift of the focal amplitude corresponds to an optical path difference of $\lambda / 2$ at the edges of the pupil (destructive interference): the center of the pupil must be bright and the pupil edges dark. The main drawback of a Michelson interferometer is that half of the flux is lost backwards to the source. However, it can be used for an easy laboratory test experiment, which will be reported in a future work. A better solution seems to use a Mach-Zendher interferometer, which has two outputs.

Obtaining two apodized pupils with a Mach-Zendher interferometer appears to be impossible for conservation of energy reasons. The relative phase shift between the reflected and transmitted beams in a thin or symmetric beam-splitter is $\pi / 2$; this imposes complementary fringes at the two outputs of the interferometer. Thus we can obtain an apodized output (with same transmission as a perfect classical apodizer) and an "anti-apodized" output which pupil amplitude is brighter at the edges and leads to the opposite effect to apodization. This output cannot directly be used for planet detection, but remains usable for other purposes, such as tracking.

The successive apodizations can be produced with several successive interferometers, but with the problem of multiplying optical surfaces. An unique multiple-beam, specifically designed interferometer may also be possible and has to be studied. Mach-Zendher interferometers made of two glass blocks associated together with total internal reflections may be preferred to mirrors to preserve a high throughput and minimize the number of optical surfaces. However, for coronagraphy, classical interferometers with mirrors would permit the user to tune the wedge fringes for practical optimization, as we shall see later.

Of course, the main drawback of such an interferometric technique is its chromatism. This point will be discussed in Sect. 5. For an actual two-dimensional aperture and if the apodization is required in two dimensions, the use of two interferometers at an orientation of $90^{\circ}$ will be needed.

\section{Analytic expressions of the PSF and corresponding apodization function}

In this section, we show that the apodization functions obtained with the interferometric technique correspond to the cosine to the power of $N$ apodizer and calculate the analytical expressions of the PSF for these apodization functions. For more generality and later use, we consider the case where the shift may be $b$ times smaller than the optimal $1 / L$ considered in the previous section.

The amplitude corresponding to the addition of two PSF images distant one another of $\frac{1}{b L}$ can be written as:

$\Psi_{b}^{(1)}(x)=\frac{1}{2} \frac{\sin \pi L x}{\pi L x} *\left(\delta\left(x-\frac{1}{2 b L}\right)+\delta\left(x+\frac{1}{2 b L}\right)\right)$

where for convenience, we use the Dirac delta function $\delta(x)$ to write the displacements between the PSF's and where the symbol $*$ denotes the convolution product. For reasons of symmetry, the two PSF's are shifted of $\frac{1}{2 b L}$ in opposite directions. This parameter $b$ is simply the factor by which the angle between the mirrors of the interferometer must be reduced. A second apodization can be obtained by again dividing $\Psi_{b}^{(1)}(x)$ into two terms and shifting them by the same amount $\pm \frac{1}{2 b L}$ as above (see Figs. 1 and 2 for illustration):

$$
\begin{gathered}
\Psi_{b}^{(2)}(x)=\frac{1}{4} \frac{\sin \pi L x}{\pi L x} *\left(\delta\left(x-\frac{1}{2 b L}\right)+\delta\left(x+\frac{1}{2 b L}\right)\right) \\
*\left(\delta\left(x-\frac{1}{2 b L}\right)+\delta\left(x+\frac{1}{2 b L}\right)\right),
\end{gathered}
$$

and we simply obtain the addition of three terms of the form:

$$
\begin{aligned}
\Psi_{b}^{(2)}(x)= & \frac{1}{4}\left(\frac{\sin \pi L x}{\pi L x}\right) \\
& *\left(\delta\left(x-\frac{1}{b L}\right)+2 \delta(x)+\delta\left(x+\frac{1}{b L}\right)\right) .
\end{aligned}
$$

We can write the resulting impulse response for the $N$ th order apodization, as follows:

$$
\begin{aligned}
\Psi_{b}^{(N)}(x)=\frac{1}{2^{N}} & \left(\frac{\sin \pi L x}{\pi L x}\right) \\
& *\left(\delta\left(x-\frac{1}{2 b L}\right)+\delta\left(x+\frac{1}{2 b L}\right)\right)^{* N}
\end{aligned}
$$


Table 1. Apodized impulse responses, for a one-dimensional aperture of width $L$.

\begin{tabular}{ll}
\hline Apodization & Impulse responses (normalized) \\
\hline unapodized: & $\frac{\sin (\pi L x)}{\pi L x}$ \\
1st order, $b=1$ & $\frac{\cos (\pi L x)}{\left(1-4 x^{2} L^{2}\right)}$ \\
2nd order, $b=1$ & $\frac{\sin (\pi L x)}{(\pi L x)\left(1-x^{2} L^{2}\right)}$ \\
3rd order, $b=1$ & $\frac{\cos (\pi L x)}{\left(1-4 x^{2} L^{2}\right)\left(1-4 x^{2} L^{2} / 9\right)}$ \\
\hline
\end{tabular}

where the symbol $* N$ means that the convolution must be done $N$ times. Alternatively, this equation can be rewritten using the binomial relation:

$$
\begin{aligned}
\Psi_{b}^{(N)}(x)=\frac{1}{2^{N}} & \left(\frac{\sin \pi L x}{\pi L x}\right) \\
& * \sum_{k=0}^{N}\left(\begin{array}{l}
N \\
k
\end{array}\right) \delta\left(x-\left(k-\frac{N}{2}\right) \frac{1}{b L}\right) .
\end{aligned}
$$

For the particular case where $b=1$, these expressions find a simple form. Depending on the parity of $N$, we have:

- For $N$ even:

$$
\begin{aligned}
\Psi_{1}^{(N)}(x) & =\frac{1}{2^{N}} \frac{\sin \pi L x}{\pi L x \prod_{k=1}^{N / 2}\left(1-x^{2} L^{2} / k^{2}\right)} \\
& =\frac{1}{2^{N}} \prod_{k=N / 2+1}^{\infty}\left(1-\frac{x^{2} L^{2}}{k^{2}}\right) .
\end{aligned}
$$

- For $N$ odd:

$$
\begin{aligned}
\Psi_{1}^{(N)}(x) & =\frac{1}{2^{N}} \frac{\cos \pi L x}{\prod_{k=1}^{(N+1) / 2}\left(1-4 x^{2} L^{2} /(2 k-1)^{2}\right)} \\
& =\frac{1}{2^{N}} \prod_{k=(N+3) / 2}^{\infty}\left(1-\frac{4 x^{2} L^{2}}{(2 k-1)^{2}}\right),
\end{aligned}
$$

where the last part of Eqs. (7) and (8) are obtained by writing the sine and cosine functions as infinite products. The interest of this last form is mainly to show that the resulting function $\Psi_{1}^{(N)}(x)$ are even functions, with a central width (defined as the distance between first zeros) equal to $(N+2) / L$. The numerical computation is easily done using the first part of Eqs. (7) and (8) and the three first orders are given in Table 1; illustrations of the intensity PSF are given in Fig. 3 for $N=0$ (no apodization) to $N=3$.

The corresponding amplitude in the pupil plane can be obtained directly as the Fourier transform of Eq. (5). Using basic properties of the Fourier transform of a convolution product, we obtain the simple expression:

$$
P_{X}^{N}(x)=\cos ^{N}\left(\frac{\pi x}{b L}\right) \times \Pi\left(\frac{x}{L}\right) .
$$
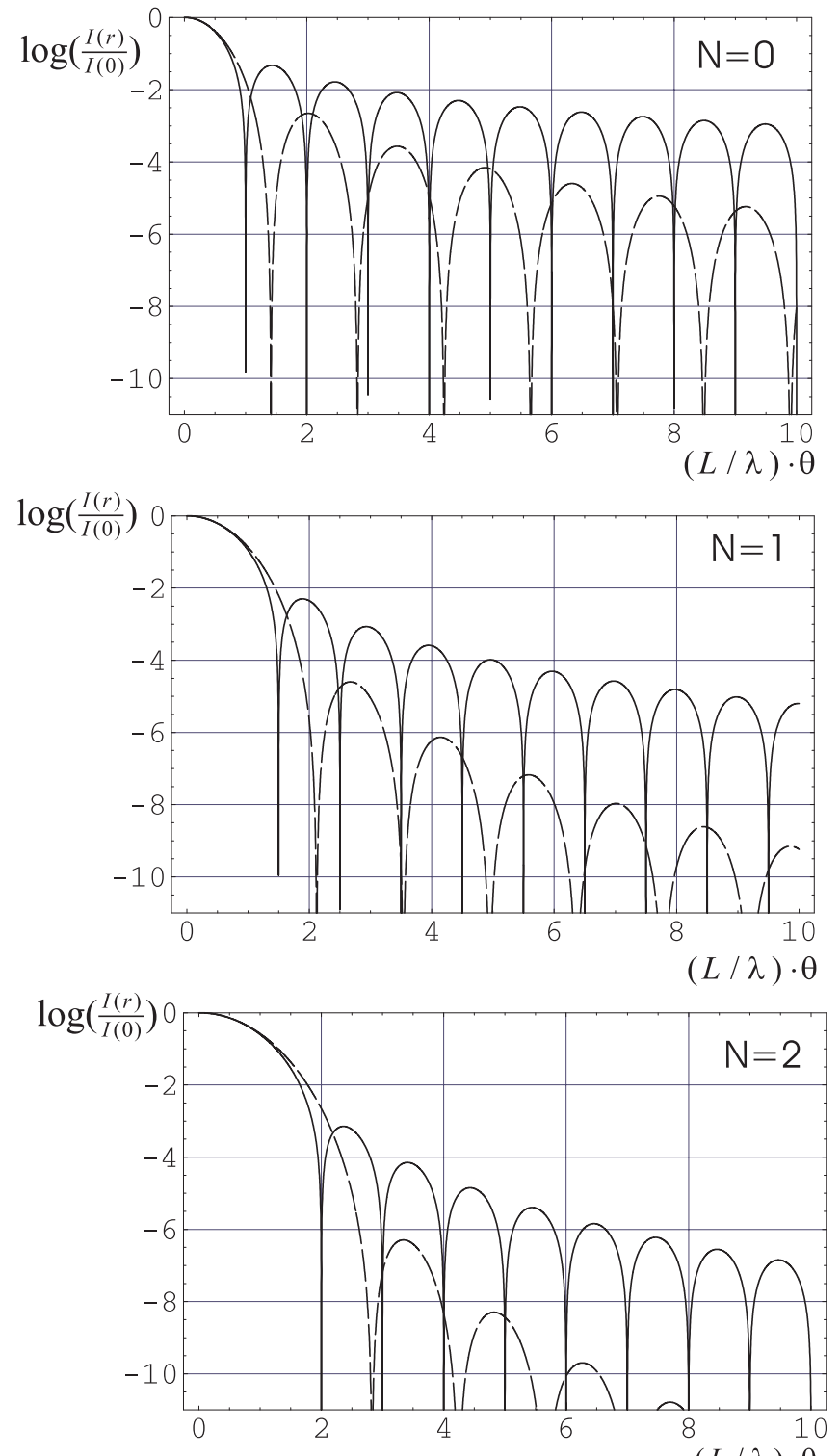

$(L / \lambda) \cdot \theta$

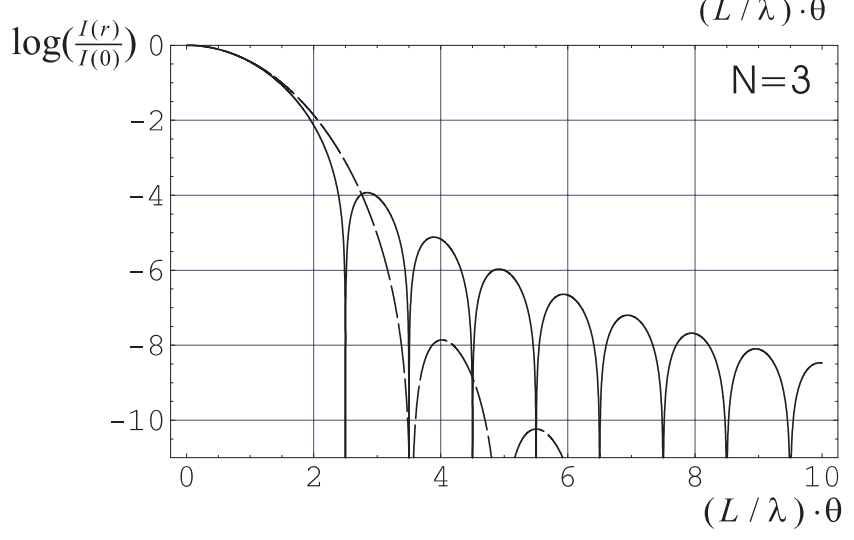

Fig. 3. From top to bottom: unapodized PSF, and successive order of $N$ given in Table 1. Dashed plots corresponds to a cut of the PSF along the diagonal direction, as considered in the ASA Concept.

For $b=1$, we recognize the cosine and cosine squared apodization functions proposed by Nisenson \& Papaliolios (2001). This result was expected since the apodization corresponds to a wedge fringe in the pupil plane. 
The proposed interferometric technique may be a solution to produce these apodization functions for the ASA concept. To obtain the two dimensional apodizer, two successive devices must be successively used in orthogonal directions. Making $b$ larger than one corresponds to the use of a partial cosine arch apodization: only the central part is used. This apodization will be of interest for coronagraphy (Sect. 4). The above expressions for the amplitude impulse response can be used for an analytical study of the ASA concept even if the interferometric technique is not used to produce the aperture transmission: the diffracted light intensity along the diagonal for a square aperture is simply written as the fourth power of the amplitudes given in Eqs. (7), (8) and Table 1. Illustrations of the diagonal diffracted light for the ASA are given in Fig. 3. These curves were already numerically given by Nisenson \& Papaliolios (2001).

\section{Application of interferometric apodization to stellar coronagraphy}

\subsection{Needs of entrance pupil apodization in stellar coronagraphy}

The purpose of coronagraphy is to reduce the star diffracted light in the whole field to provide direct imaging capabilities of faint sources in the vicinity of the star. For the sake of clarity, we briefly recall some fundamental aspects of this otherwise very well-known technique.

In Lyot's coronagraphy (Lyot 1930) applied to exoplanet detection, an opaque mask is set at the center of the stellar image. According to Babinet's theorem, the diffraction pattern of this dark mask appears in negative amplitude on an image of the entrance-pupil. There, a diaphragm (Lyot's stop) is set to get rid of the light diffracted outside the telescope aperture image. Inside this diaphragm, direct and diffracted light of the star are interfering destructively, while the light coming from the planet remains almost unaffected. However, because the smooth diffraction pattern of the mask cannot fit the flat impinging wavefront, the starlight is not fully cancelled. Typically, an integrated reduction factor of the order of $10^{-3}$ may be obtained using an optimized Lyot mask for a square aperture. The technique was improved by Roddier \& Roddier (1997) and Guyon et al. (1999), replacing the opaque mask by a $\pi$ Phase Mask (PM): the performance can be enhanced while the principle remains the same.

Most of the star residual light appears at the edges of the pupil in the relay pupil plane. In the original technique, the Lyot's stop diameter is smaller than the pupil size to reduce this effect. A classical improved solution is to use optimized entrance pupil apodization. We examine here the effect of the apodized apertures permitted by the technique proposed in the previous section.

For a rectangular aperture of size $L_{x} \times L_{y}$, the coronagraphic mask must be a rectangle of size $a_{x} \times a_{y}$ proportional to the inverse of the aperture. Following the approach of Guyon \& Roddier (2000) and Aime et al. (2001), the residual amplitude within the entrance aperture left by the coronagraphic process can be written as:

$\Psi(x, y)=P_{X}^{N}(x) P_{Y}^{N}(y)-\varepsilon F_{X}(x) F_{Y}(y)$

where $\varepsilon=1$ for Lyot's coronagraphy and $\varepsilon=2$ for Roddier's coronagraphy and $P_{X}^{N}(x)$ is the entrance apodized pupil of Eq. (9). The function $F(x)$ is the convolution product of the aperture and the diffraction pattern of the mask:

$F_{X}(x)=P_{X}^{N}(x) * \frac{\sin \pi a_{x} x}{\pi x}$.

Analytic expressions for $F_{X}(x)$ are given in Appendix A. Corresponding values of $a_{x}, a_{y}$ and $b_{x}, b_{y}$ depend on the sizes of $L_{x}$ and $L_{y}$.

The case for entrance pupil apodization combined with coronagraphy appears clearly in Eq. (10): the idea is to optimize the pupil shape to achieve the best amplitude subtraction for the on-axis unresolved star. In other words we shall try to solve the equation $\Psi(x, y)=0$ within the aperture.

This solution can be approached very closely by using an iterative numerical algorithm as first proposed by Guyon \& Roddier (2000): iterations are computed between the entrance and exit pupil planes, and the residual amplitude is negatively re-injected in the entrance pupil. The apodization functions so obtained do not resemble to the full cosine apodized aperture $(b=1)$, but are very similar to the central part of a cosine arch.

We recently noticed that there is a formal solution to this problem (Aime et al. 2001, submitted) and that the cosine arch is indeed a very good approximation to a perfect apodization. In any case, as we shall see below, it permits the aperture to give very good stellar light rejection.

\subsection{Residual energy for Lyot's and R\&R's optimized apodized rectangular apertures.}

We consider here a square aperture for which $a_{x}=a_{y}=a$ and $b_{x}=b_{y}=b$. A simple optimization criterion to test the efficiency of the coronagraphic experiment is to consider the integrated residual energy within the exit pupil, for the unresolved on-axis star. Note that since coronagraphy is a direct imaging technique, this criterion is rather pessimistic. Using minimisation techniques, the optimal mask size $a$ and apodization parameter $b$ can be numerically computed. The results are given in Table 2 for a square aperture, and a few details on the computations are given in Appendix B.

It may be also interesting to represent the variations of this energy as a two dimensional function of $a$ and $b$. This is done in Fig. 4, for a square aperture, and may be more visible in Fig. 5 where slices of the functions of Fig. 4 are given.

Lyot's and R\&R's techniques have quite different behaviours: there is a single well-defined global minimum for 

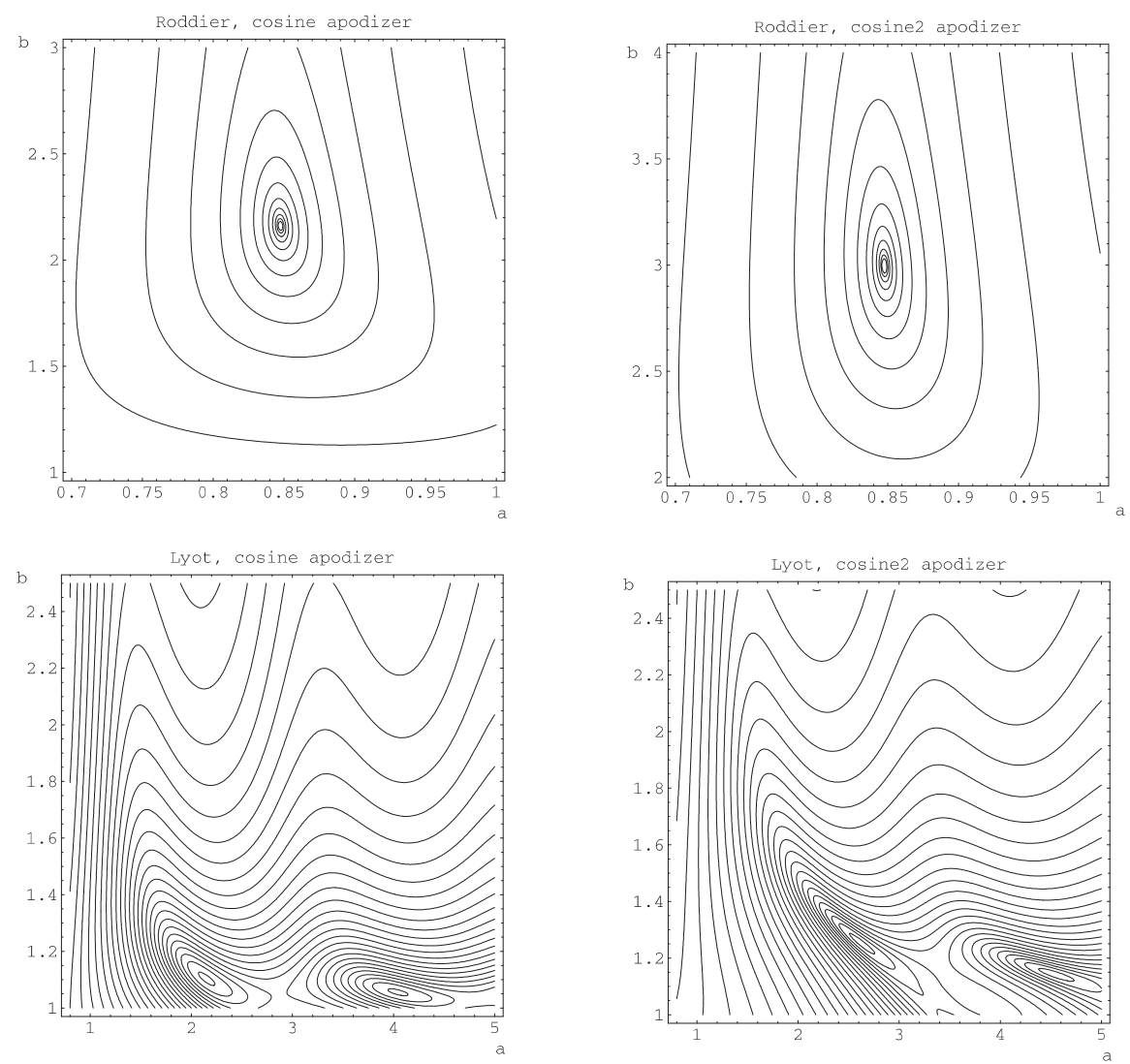

Fig. 4. Residual energy as a function of the mask size $a$ and apodization parameter $b$ for a square aperture. The value $b=$ 1 corresponds to full cosine apodization (Sect. 2). Higher values of $b$ correspond to a partial apodization. For Lyot's coronagraphy several minima are obtained for different $a$ and $b$ values. See Table 2 for numerical values.

Table 2. Results of the analytical optimization for cosine apodizers and coronagraphy.

\begin{tabular}{lllll}
\hline Technique & $\begin{array}{l}\text { Apodization } \\
\text { parameter: } b\end{array}$ & $\begin{array}{l}\text { Mask size: } \\
a \times L\end{array}$ & $\begin{array}{l}\text { Residual energy } \\
\text { (square aperture) }\end{array}$ & $\begin{array}{l}\text { Apodizer intensity transmission } \\
\text { (square aperture) }\end{array}$ \\
\hline R\&R, $\cos (N=1)$ & 2.160 & 0.848 & $E=1.14 \times 10^{-6}$ & $70 \%$ \\
R\&R, $\cos ^{2}(N=2)$ & 2.995 & 0.848 & $E=3.30 \times 10^{-8}$ & $70 \%$ \\
Lyot, $\cos (N=1)$ & 1.103 & 2.147 & $E=6.67 \times 10^{-4}$ & $30 \%$ \\
Lyot, $\cos ^{2}(N=2)$ & 1.254 & 2.621 & $E=2.95 \times 10^{-5}$ & $22 \%$ \\
Lyot 2nd min. $(N=1)$ & 1.056 & 4.040 & $E=1.51 \times 10^{-4}$ & $30 \%$ \\
Lyot 2nd min. $(N=2)$ & 1.154 & 4.468 & $E=4.15 \times 10^{-6}$ & $19 \%$ \\
ASA $\cos (N=1)$ & 1 & & & $25 \%$ \\
ASA $\cos ^{2}(N=2)$ & 1 & & & $14 \%$ \\
\hline
\end{tabular}

the PM technique, whereas successive several local minima exist for Lyot's coronagraphy as the mask size increases. The optimal $b$ value is different if the solution is searched at the first or second minimum. This means that for a given shape of apodization, there is an optimal mask size, and is reciprocally so. The second optimal value gives a much better extinction ratio, at the expense of a reduced capability of detecting a planet very close to the star (larger $a$ ). These effects are visible in Figs. 4, 5 and in Table 2.

Since most of the residual light is concentrated at the edges of the exit pupil, even with entrance pupil apodization, we can take advantage of the initial Lyot's method and use a smaller exit pupil than the entrance pupil. We have empirically tested a few Lyot's stop reductions $(90 \%, 80 \%)$. The optimization results are different when carried out with a reduced Lyot's stop and can provide a better extinction inside the reduced exit pupil. However, a detailed study of the Signal to Noise Ratio (SNR) would be needed to determine the optimum exit pupil reduction.

A representation of the residual intensities $|\Psi(x, y)|^{2}$ of Eq. (10) is given in Fig. 6 for the optimal values of $a$ and $b$. The residual field intensity (RFI) for the optimal cosine apodizations with coronography are illustrated in Fig. 7. These RFI expressions are not analytic and have 

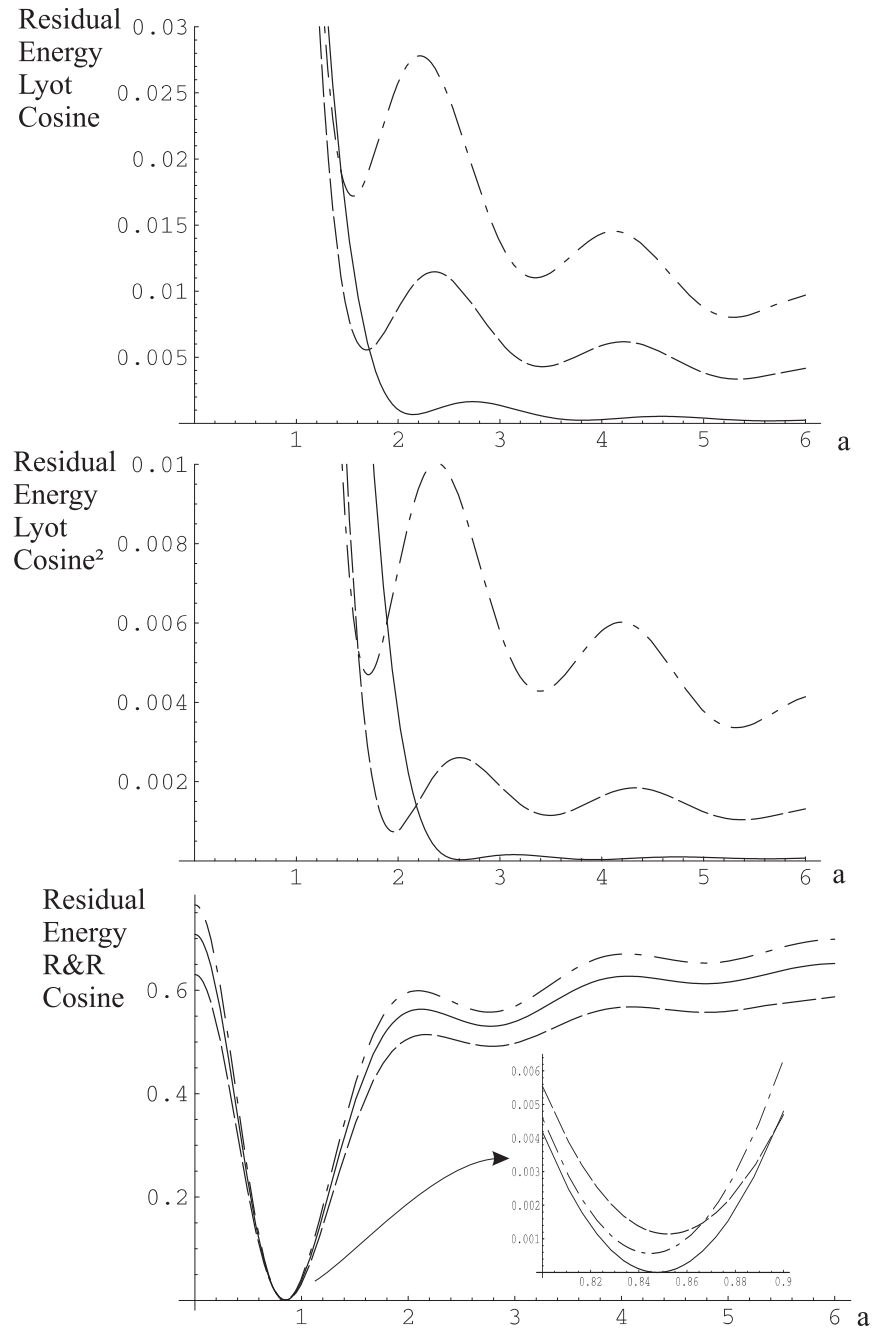

Fig. 5. Slices of the energy for different values of apodization parameter $b$ (full lines correspond to the optimal b of Table 2).

been computed numerically. The results, summarized in Table 2, lead to the following comments:

- For R\&R's coronagraphy with a cosine apodization, a very low residual integrated energy of $1.14 \times 10^{-6}$ for a two dimensional aperture can be obtained with the cosine apodization $P_{X}^{1}(x)$ and the values $a=0.848 / L$ and $b=2.160$. The transmission of this apodizer is of $70 \%$ for a square aperture. A cosine squared $P_{X}^{2}(x)$ apodization gives better results for about the same transmission and the residual energy is down to $3.30 \times 10^{-8}$.

- For Lyot's coronography the results are given for the two first local minima of the residual energy. The use of further minima is possible but leads to a prohibitive reduction of the field of view. As it can be seen from the values of $b$ of Table 2 and illustrations, this technique requires a much stronger apodization than R\&R's, close to what is needed for ASA. The residual energy for cosine squared apodization with $P_{X}^{2}(x)\left(E=2.95 \times 10^{-5}\right)$ gives much better results than $P_{X}^{1}(x)\left(E=6.67 \times 10^{-4}\right)$.
Lyot's technique is however much less efficient than $R \& R$ 's one in terms of resolution since the pupil mask is much larger. However the technique may have interesting aspects: it is much simpler, and performances of the coronagraph are not very sensitive to the preciseness of $a$. Lyot's technique might eventually be used at the second minimum leading of course to a higher rejection, comparable to that obtained with a PM with cosine apodization, but the capability of detecting a close-by planet is about 5 times worse than for the $\mathrm{R} \& \mathrm{R}$ technique.

\subsection{PSF for the planet}

A PSF for the planet can be computed if we assume that the coronagraphic mask (Lyot's mask or R\&R's phase mask) has almost no effect on the re-imaged planet whatever its position in the field. For that we can use Eq. (2) and Eq. (3) i.e. the sum of two or three shifted sine cardinal functions. An illustration is given in Fig. 9. Note that here the smaller the PSF, the better the result, since the detection of the planet will be made with less background noise.

\section{Chromatic effects: Discussion on the possibility of broad-band observations}

The interferometric technique we have described in Sect. 2 to obtain the cosine apodizations is wavelength sensitive. Since the size of the diffraction pattern in the focal plane increases with the wavelength, shifts and mask sizes should vary accordingly. If not, and such is the case that we analyse here, the parameters $a$ and $b$ are only optimized for a single wavelength $\lambda_{0}$ within the bandwidth.

The computations made previously can be used to compute chromatic effects. For that, we simply assume that at a wavelength $\lambda$ we are using inappropriate $a(\lambda)$ and $b(\lambda)$ values of the form:

$b(\lambda)=\frac{\lambda}{\lambda_{0}} b_{0}, \quad a(\lambda)=\frac{\lambda_{1}}{\lambda} a_{1}$

where $a_{0}$ and $b_{0}$ are the optimal values at $\lambda_{0}$.

For pure apodization, the resulting PSF is obtained integrating $\left|\Psi_{b}^{(N)}(x)\right|^{2}$ of Eq. (5) within the spectral window:

$I_{\Delta \lambda}(x)=\int_{\lambda}^{\lambda+\Delta \lambda}\left|\Psi_{b(\lambda)}^{(N)}(x)\right|^{2} \mathrm{~d} \lambda$.

In that relation, $b(\lambda)=\lambda / \lambda_{0}$ since $b_{0}=1$ (pure apodization). Examples of the results for different spectral windows are shown in Fig. 10. The chromatic effect degrades the result, as expected, but it is not as significant as one could fear. The curves can be compared to the monochromatic simulations in Fig. 3.

A solution to the chromatic problem would be to use an achromatization lens-system which produces a magnification of the focal image proportional to $1 / \lambda$ over the 

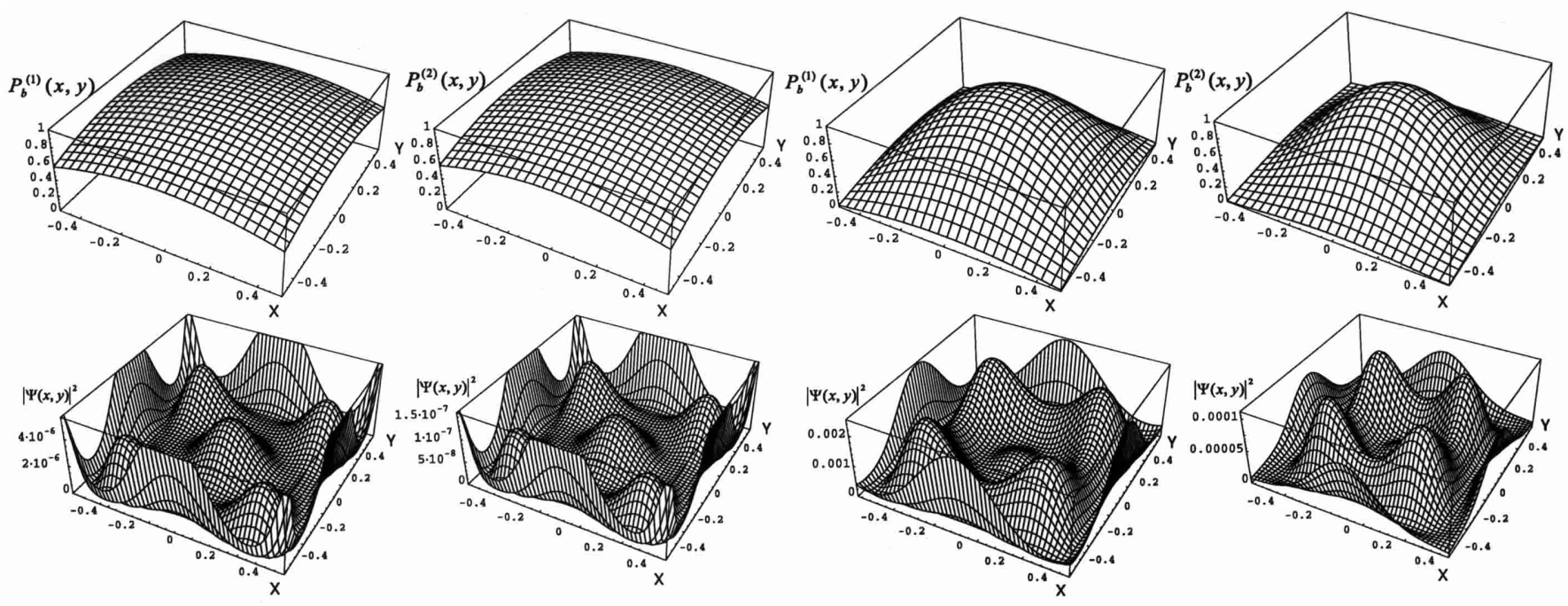

Fig. 6. Top: entrance apodized pupils (amplitude transmission). Bottom: residual intensity in the exit pupil plane. From left to right: $\mathrm{R} \& \mathrm{R}$ with cosine, $\mathrm{R} \& \mathrm{R}$ with cosine squared, Lyot with cosine, Lyot with cosine squared.
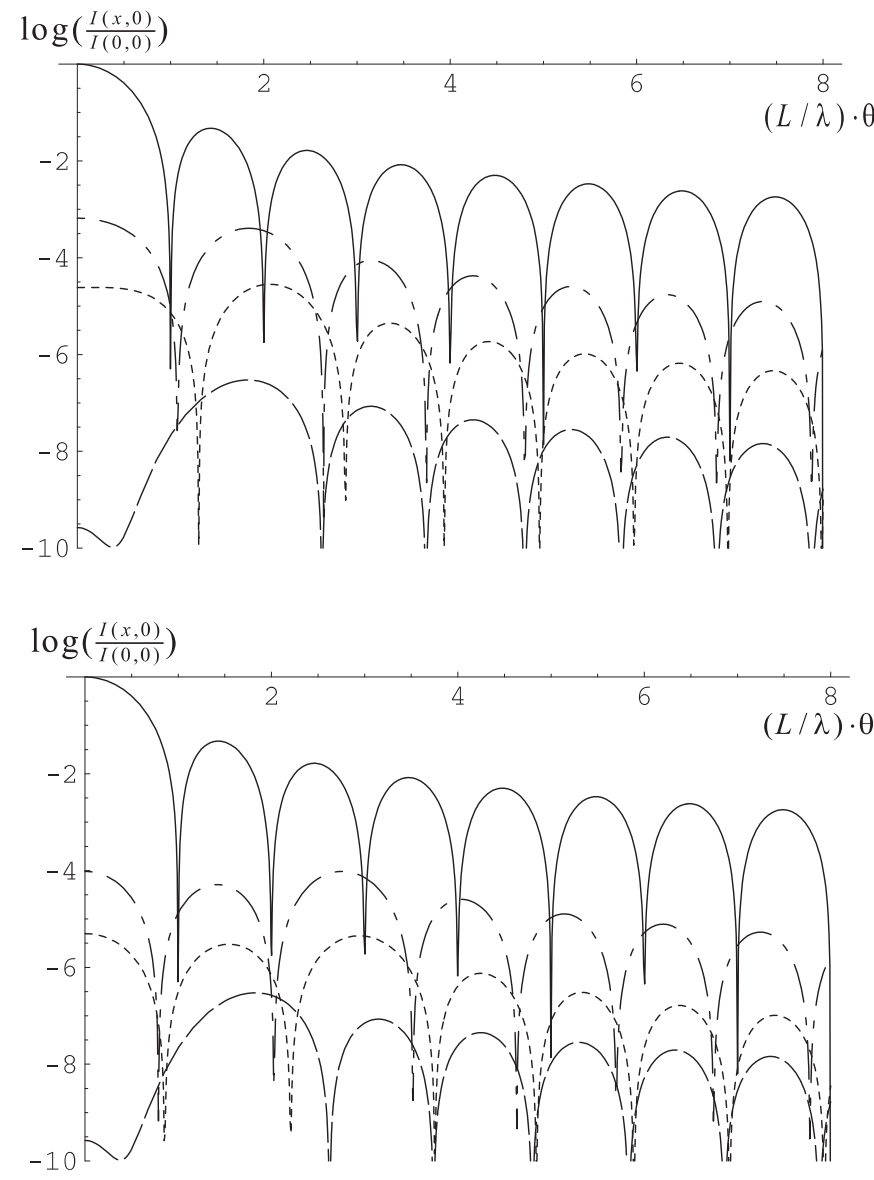

Fig. 7. Residual Field Intensity in the final focal plane for a one-dimensional aperture. Top: PSF for a raw aperture without apodization nor coronagraphy (full line), Lyot's coronagraphy with a cosine apodizer (dash-dotted). Lyot's coronagraphy with a cosine squared apodizer (dotted). R\&R's coronagraphy with a cosine apodizer (long-dashed)). Bottom: Lyot's coronagraphy is plotted at the second minimum (see Table 2 for the corresponding parameters). bandpass. These kind of achromatizers have been successfully tested for pupil plane interferometry (Wynne 1979; Roddier et al. 1980). In that case, the image of the planet will appear as a dispersed spectrum. For very wide bandwiths, such an achromatization technique would probably fail and a separation into several bands is certainly needed to adapt the achromatization in each band.

On the other hand, classical apodizers using absorbing devices also present technological challenges to realize the exact transmission function. The physical absorption process is wavelength dependent, and chromatic problems may also occur; multilayers may be needed to obtain an achromatic transmission over a wide bandpass. With the interferometric technique, the apodization function (cosine, cosine squared) is physically produced by the interference phenomenon itself and may be exactly obtained.

For PM coronagraphy, the first difficulty is to obtain a wavelength independent $\pi$ phase mask. Assuming that this result is obtained, we can compute the effect relative to the variation of the parameters $a$ and $b$ within the spectral bandwidth. Curves of Fig. 4 can be used for that: the residual intensity is the result of the integration along the parametric curve defined by equations $a(\lambda)$ and $b(\lambda)$ of Eq. (12). Examples are given in Fig. 11, for the one-dimensional case, where we consider that a perfect $\pi$ phase mask is used.

The chromatic effect on the mask size is much more sensitive in the $R \& R$ case than in Lyot's case. This can be understood easily from Fig. 5. R\&R's coronagraphy may give even worse results than Lyot if the chromatic effect on the mask size is not corrected. On the contrary, R\&R coronagraphy is not very sensitive concerning the chromatism of the apodization, because the optimal apodization function corresponds to a very weak apodization (partial cosine arch) 

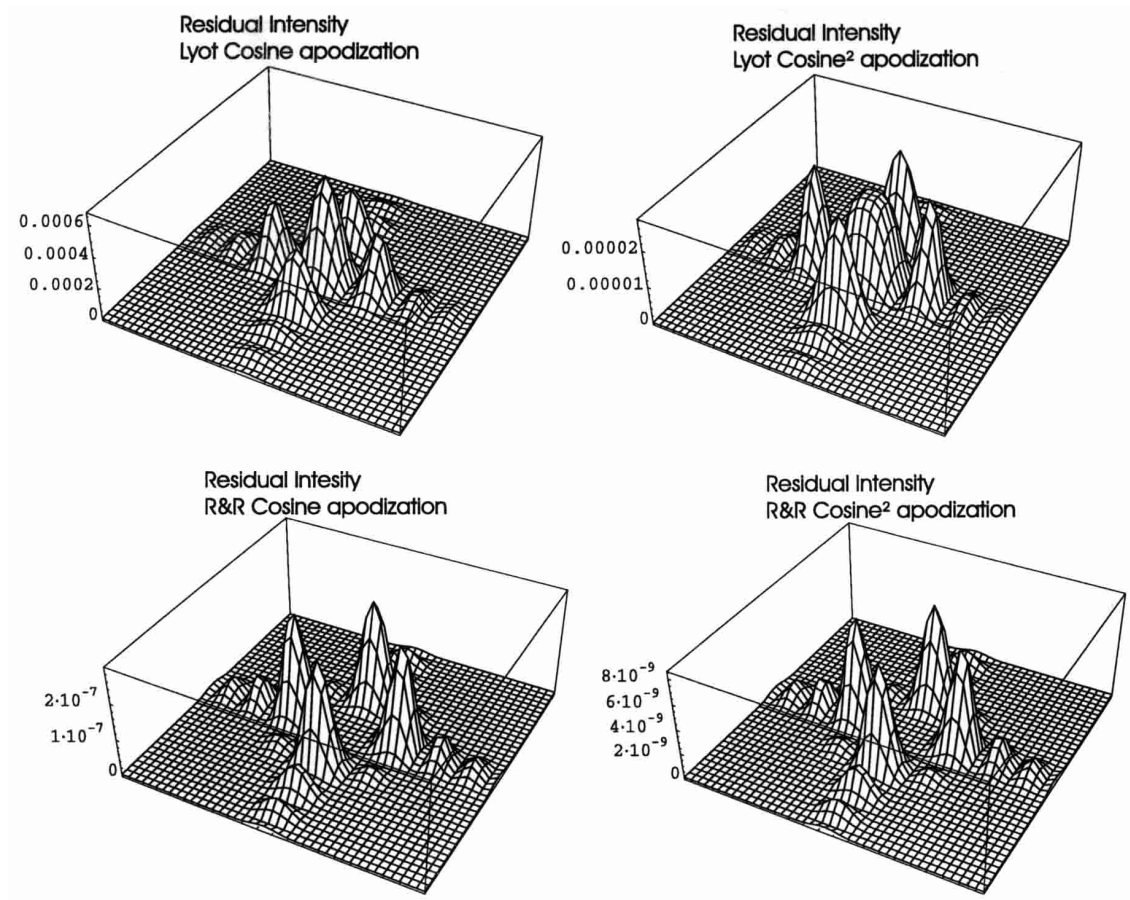

Fig. 8. Residual intensity of the star in the focal plane for the 4 considered techniques: Top left: Lyot's coronagraphy with cosine apodization (at first minimum). Top right: Lyot's coronagraphy with cosine squared apodization (at first minimum). Bottom Left: R\&R's PM with cosine apodization. Bottom right: R\&R's PM with cosine squared apodization.

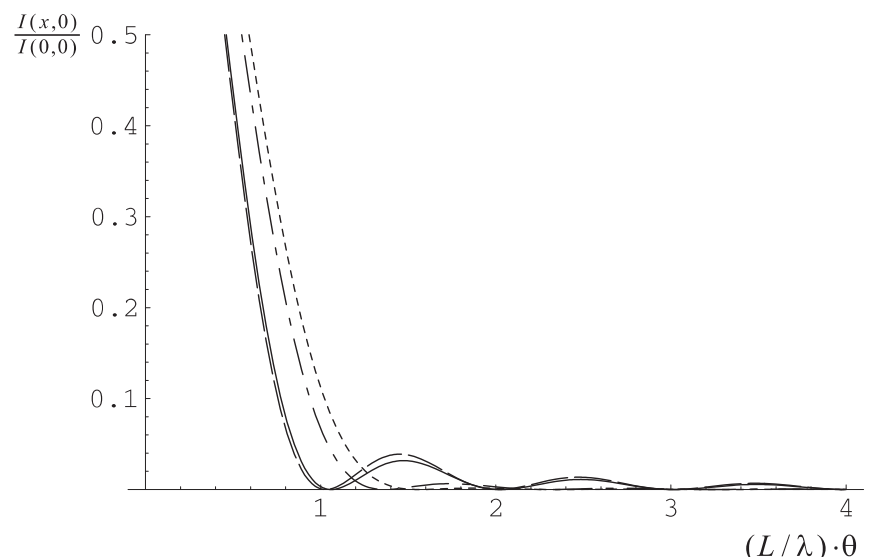

Fig. 9. PSF for the planet (the effect of coronagraphy on the off-axis planet is assumed to be negligible), PSF for R\&R with a cosine apodization (full line), PSF for R\&R with a cosine squared apodization (long dash), PSF for Lyot with a cosine apodization (dashed line), PSF for Lyot with a cosine square apodization (dotted line)

\section{Conclusion}

We have proposed in this paper a simple apodization technique for rectangular or square apertures that can be implemented using classical optical devices. It consists of splitting the focal image in two parts that are shifted and added coherently, so that maxima and minima of the oscillatory part of the diffraction pattern cancel each other. This is equivalent to the use of an aperture with a cosine apodization that can be obtained using a Michelson or a Mach-Zender interferometer operated with a small angle between the mirrors. By using several interferometers,
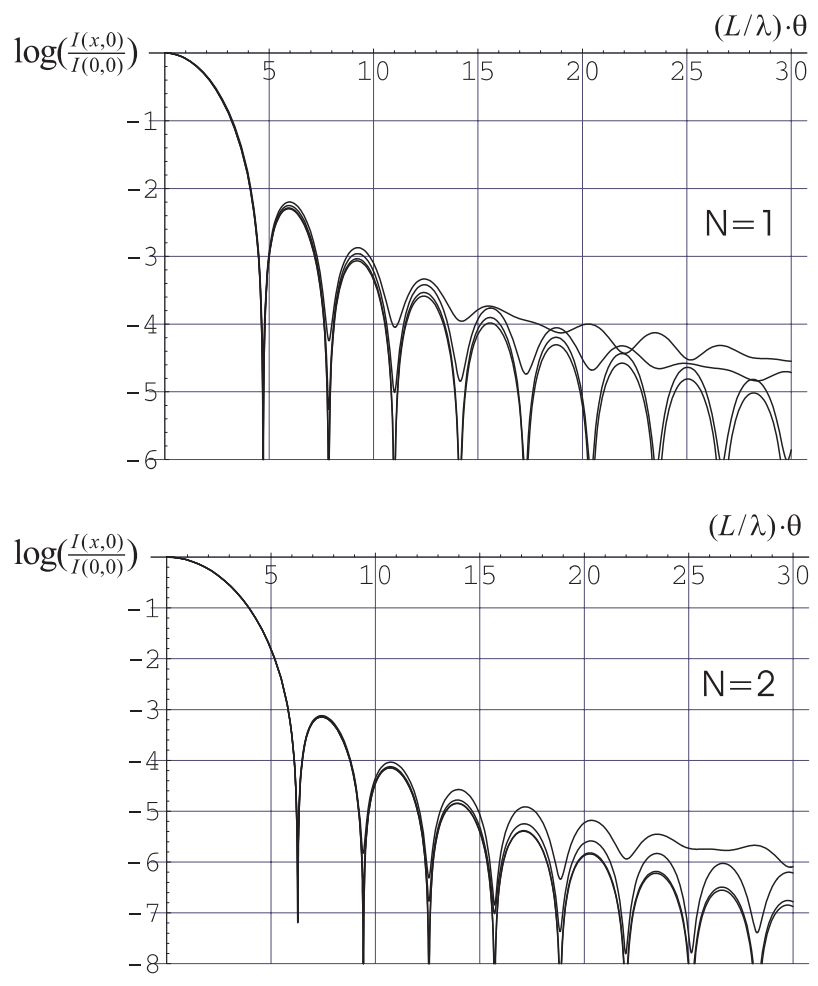

Fig. 10. Effect of increasing bandwidth on the PSF for the interferometric apodization $(N=1)$ and $(N=2)$ : achromatic PSF, effect of a $10 \%$ bandwidth, $20 \%$ bandwidth, $30 \%$ bandwidth.

any integer power of cosine functions can be obtained. The apodization is done in one direction (along a side of the rectangle). Two interferometers set in orthogonal directions are needed to apodize the rectangular aperture in the two directions. 

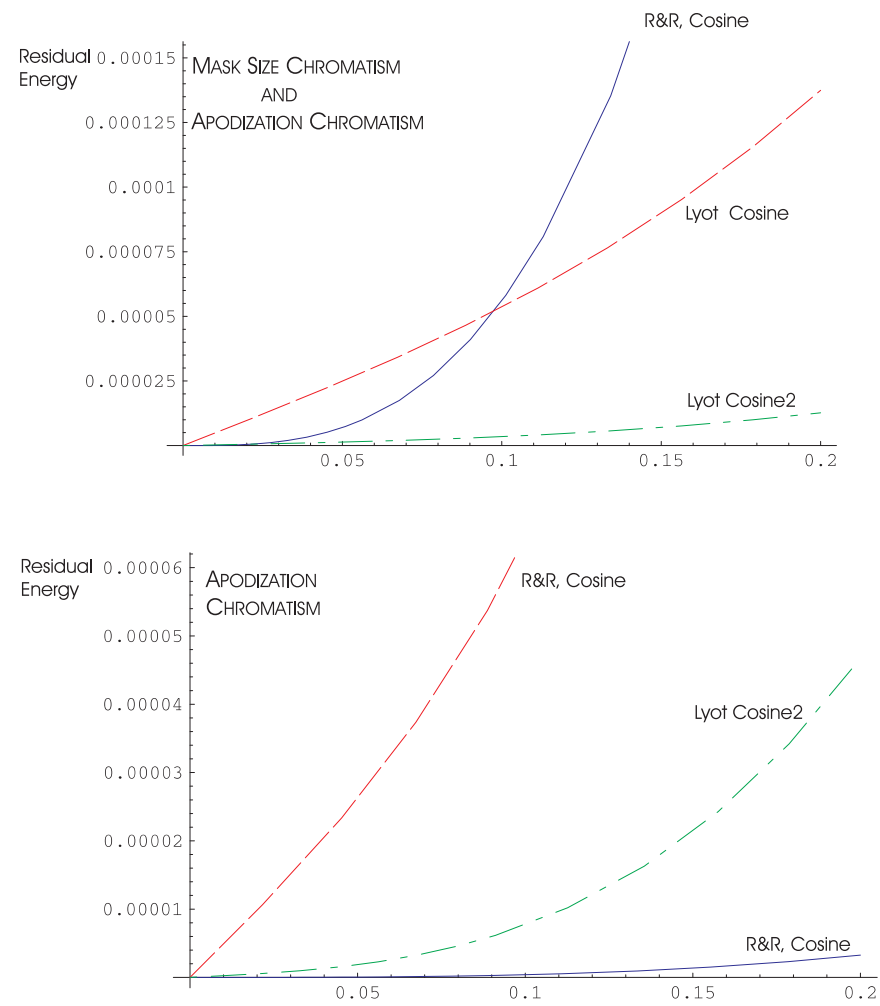

Fig. 11. Residual energy as a function of the bandwith expressed in percents (this computation is made for the onedimensional case). Top: effect of mask size chromatism and interferometric apodization chromatism. $\mathrm{R} \& \mathrm{R}$ is much more sensitive to the mask size and can be worse than Lyot for a bandwidth over 10\%. Bottom: effect of interferometric apodization alone (mask size chromatism corrected). The $R \& R$ 's technique is less sensitive to the chromatism of the apodizer.

The technique is then studied for application to the ASA concept recently proposed by Nisenson \& Papaliolios (2001). Our approach allowed us to give a simple analytic expression for the cosine diffraction patterns considered by these authors. For a cosine to the power $N$ apodization, the resulting focal plane amplitude is simply written as the sum of $N+1$ weighted and shifted sine cardinal functions. The weights are given by Pascal's triangle. The shifts must be equal to $1 / L$ ( $L$ being the length of the aperture in units of wavelenth) for a full cosine-arch apodized-aperture (maximum at the center and zero at the edges). In that case, very simple analytical expressions can be worked out. Making the shifts b times smaller, one obtains a partially apodized aperture (central part $1 / b$ of the cosine-arch).

We have then analyzed the effects of apodizations on coronagraphy. Here also we have been able to derive analytic expressions for the residual amplitude left in an image of the aperture after the coronagraphic experiment. We found that the full apodized aperture $(b=1)$ is not suitable for coronagraphy. On the other hand, very good results can be obtained with partial cosine apodizations. For
$R \& R$ 's phase mask technique a rejection of $1.1 \times 10^{-6}$ is obtained using a simple cosine apodization with $b=2.160$, and a rejection of $3.3 \times 10^{-8}$ is obtained using a cosine squared apodization with $b=2.995$.

The computation was made for a square aperture, but it could also apply to a rectangular aperture. For Lyot's technique, the cosine apodization is not very good, and a cosine squared is needed $\left(10^{-5}\right.$ rejection with $\left.b=1.254\right)$.

It was beyond the scope of this paper to give the relative factors of merit of the ASA technique to the Lyot and $\mathrm{R} \& \mathrm{R}$ phase mask coronagraphic techniques; a major point will probably be the technical possibility of realization of each of them. The behavior depends also on the distance of the planet to the star, coronagraphic techniques being more effective for very close objects. If this distance is large, the disparities between the techniques is reduced. The proposed interferometric apodization technique appears to be versatile and can be adjusted to produce the partial or full cosine arch apodizations required by these techniques. The effect of its chromatism is not too severe for a reasonable bandwidth.

\section{Appendix A: Analytical expressions of the residual pupil amplitudes for cosine apodizers}

The residual amplitude left in the entrance aperture by the coronagraphic process (Eq. (10)) depends on the function $F(x)$ (Eq. (11)) which describes the diffraction pattern of the coronagraphic mask.

- For a raw unapodized aperture $P(x)=\Pi(x / L)$, one obtains (Aime et al. 2001):

$$
F(x)=\frac{1}{\pi}\left(\operatorname{Si}\left[\pi a\left(x+\frac{L}{2}\right)\right]-\operatorname{Si}\left[\pi a\left(x-\frac{L}{2}\right)\right]\right) .
$$

- For the partial cosine apodized aperture $\Pi\left(\frac{x}{L}\right) \cos \left(\frac{\pi x}{b L}\right)$ we have:

$$
\begin{aligned}
& F(x)= \\
& \quad \frac{-1}{2 \pi}\left(\frac{\sin (\pi x)}{b L}(-\mathrm{Ci}[A]+\mathrm{Ci}[B]+\mathrm{Ci}[C]-\mathrm{Ci}[D])\right. \\
& \left.+\frac{\cos (\pi x)}{b L}(-\mathrm{Si}[A]-\mathrm{Si}[B]+\mathrm{Si}[C]+\mathrm{Si}[D])\right)
\end{aligned}
$$

with:

$$
\begin{aligned}
& A=\pi\left(a-\frac{1}{b L}\right)\left(x-\frac{L}{2}\right), \\
& B=\pi\left(a+\frac{1}{b L}\right)\left(x-\frac{L}{2}\right), \\
& C=\pi\left(a-\frac{1}{b L}\right)\left(x+\frac{L}{2}\right), \\
& D=\pi\left(a+\frac{1}{b L}\right)\left(x+\frac{L}{2}\right) .
\end{aligned}
$$


- For the partial cosine squared apodization: $\Pi\left(\frac{x}{L}\right) \cos ^{2}\left(\frac{\pi x}{b L}\right)$, we obtain:

$$
\begin{aligned}
& F(x)= \\
& \frac{-1}{4 \pi}\left(\frac{\sin (2 \pi x)}{b L}(-\mathrm{Ci}[A]+\mathrm{Ci}[B]+\mathrm{Ci}[C]-\mathrm{Ci}[D])\right. \\
& \left.+\frac{\cos (2 \pi x)}{b L}(\mathrm{Si}[A]+\mathrm{Si}[B]+\mathrm{Si}[C]+\mathrm{Si}[D])\right) \\
& \quad+\frac{\varepsilon}{2}\left(\operatorname{Si}\left[a \pi\left(\frac{L}{2}-x\right)\right]+\operatorname{Si}\left[a \pi\left(\frac{L}{2}+x\right)\right]\right)
\end{aligned}
$$

with:

$$
\begin{aligned}
& A=\pi\left(a-\frac{2}{b L}\right)\left(x-\frac{L}{2}\right), \\
& B=\pi\left(a+\frac{2}{b L}\right)\left(x-\frac{L}{2}\right), \\
& C=\pi\left(a-\frac{2}{b L}\right)\left(x+\frac{L}{2}\right), \\
& D=\pi\left(a+\frac{2}{b L}\right)\left(x+\frac{L}{2}\right) .
\end{aligned}
$$

In these expressions, we have used the sine integral $\mathrm{Si}(x)$ and cosine integral $\mathrm{Ci}(x)$ functions defined by:

$$
\begin{aligned}
& \operatorname{Si}(x)=\int_{0}^{x} \frac{\sin t}{t} \mathrm{~d} t \\
& \mathrm{Ci}(x)=-\int_{|x|}^{\infty} \frac{\cos t}{t} \mathrm{~d} t .
\end{aligned}
$$

\section{Appendix B: Notes on the numerical computations}

The computations have been carried out analytically as far as possible and we have used the software Mathematica (Wolfram 1999). However, some of the results have been obtained numerically.

- The residual intensity $|\Psi(x, y)|^{2}$ (computed with Eq. (10)) cannot be integrated analytically to obtain the residual star energy, and the integration must be performed numerically. We have used a Gauss-Kronrod integration algorithm provided by Mathematica.

- The optimal parameters $a$ and $b$ have been obtained with a numerical minimization of the residual energy criterion, using a conjugate gradient method.

- The Fourier transform to compute the final residual intensities for the star can be separated into a difference of one-dimensional computations. If we consider the spatial limitation of the Lyot's stop and the symmetry properties of the residual wave amplitude, the Fourier integral can be favorably computed directly within the finite exit pupil, with only the cosine terms and using a numerical integration algorithm.

Acknowledgements. The authors would like to thank François Martin for his very helpful contribution to the preliminary laboratory tests of the interferometric apodization technique. Thanks are also due to François-Xavier Schmider for interesting discussions.

\section{References}

Abe, L., Vakili, F., \& Boccaletti, A. 2001, A\&A, 374, 1161

Aime, C., Soummer, R., \& Lopez, B. 2001, A\&A, 370, 680

Angel, J. R. P., \& Woolf, N. J. 1997, ApJ, 475, 373

Baudoz, P., Rabbia, Y., \& Gay, J. 2000a, A\&AS, 141, 319

Baudoz, P., Rabbia, Y., Gay, J., et al. 2000b, A\&AS, 145, 341

Boccaletti, A., Riand, P., Moutou, C., \& Labeyrie, A. 2000, Icarus, 145, 628

Bonneau, D., Josse, M., \& Labeyrie, A. 1975, in ASSL vol. 54: Image Processing Techniques in Astronomy, 403

Bracewell, R. N. 1978, Nature, 274, 780

Gay, J., \& Rabbia, Y. 1996, C. R. Acad. Sci Paris, 322 Sér. II b, 265

Guyon, O., Roddier, C., Graves, J. E., et al. 1999, PASP, 111, 1321

Guyon, O., \& Roddier, F. 2000, in Proc. of SPIE, vol. 40006

Harris, F. J. 1978, Proc. IEEE, 66, 12

Jacquinot, P., \& Roizen-Dossier, B. 1964, Progress in Optics, ed. E. Wolf, vol. 3

Leger, A. 1993, The Darwin Mission Concept, proposal to the ESA Horizon 2000 planning process

Leger, A., Mariotti, J., Mennesson, B., et al. 1999, A\&A, 341, 304

Lyot, B. 1930, C. R. Acad. Sci Paris, 191, 834

Lyot, B. 1939, MNRAS, 99, 580

Malbet, F. 1996, A\&AS, 115, 161

Mennesson, B., \& Mariotti, J. 1997, Icarus, 128, 202

Nisenson, P., \& Papaliolios, C. 2001, ApJ, 548, L201

Papoulis, A. 1981, Signal Analysis (ed. M. G. Hill)

Pedretti, E., Labeyrie, A., Arnold, L., et al. 2000, A\&AS, 147, 285

Roddier, C., Roddier, F., Martin, F., Baranne, A., \& Brun, R. 1980, Journal of Optics, 11, 149

Roddier, F., \& Roddier, C. 1997, PASP, 109, 815

Rouan, D., Riaud, P., Boccaletti, A., Clénet, Y., \& Labeyrie, A. 2000, PASP, 112, 1479

TPF Science Working Group. 1999, The Terrestrial Planet Finder TPF, ed. C. Beichman, N. Woolf, \& C. Lindensmith (http://tpf.jpl.nasa.gov/)

Watson, S. M., Mills, J. P., Gaiser, S. L., \& Diner, D. J. 1991, Appl. Opt., 30, 3253

Wolfram, S. 1999, The Mathematica book, Fourth Ed. (Cambridge university press)

Wynne, C. G. 1979, Opt. Commun., 28, 21 